\title{
Jacques Lemarchand, Le Nouveau théâtre (1947-1968). Un combat au jour le jour
}

Paola Perazzolo

\section{(2) OpenEdition}

Journals

\section{Edizione digitale}

URL: http://journals.openedition.org/studifrancesi/6088

DOI: $10.4000 /$ studifrancesi.6088

ISSN: 2421-5856

Editore

Rosenberg \& Sellier

\section{Edizione cartacea}

Data di pubblicazione: 1 mai 2011

Paginazione: 210

ISSN: 0039-2944

\section{Notizia bibliografica digitale}

Paola Perazzolo, «Jacques Lemarchand, Le Nouveau théâtre (1947-1968). Un combat au jour le jour»,

Studi Francesi [Online], 163 (LV | I) | 2011, online dal 30 novembre 2015, consultato il 11 janvier 2021

URL: http://journals.openedition.org/studifrancesi/6088; DOI: https://doi.org/10.4000/studifrancesi. 6088

Questo documento è stato generato automaticamente il 11 janvier 2021.

\section{(c) (i) $\odot$}

Studi Francesi è distribuita con Licenza Creative Commons Attribuzione - Non commerciale - Non opere derivate 4.0 Internazionale. 


\title{
Jacques Lemarchand, Le Nouveau théâtre (1947-1968). Un combat au jour le jour
}

\author{
Paola Perazzolo
}

\section{NOTIZIA}

JACQUES LEMARCHAND, Le Nouveau théâtre (1947-1968). Un combat au jour le jour, édition de Véronique HOFFMANN MARTINOT, Paris, Gallimard, 2009 («Les Cahiers de la NRF»), pp. 448.

1 Introdotto da uno scritto di Robert Abirached, il presente volume riunisce una selezione di resoconti redatti dal critico di «Combat», della «NRF» e del «Figaro littéraire» nel corso del ventennio in cui drammaturghi quali Ionesco, Adamov, Beckett, Genet, Schéhadé, ma anche Duras, Vauthier, Audiberti, Vinaver e uno riscoperto Ghelderode rinnovano profondamente il panorama teatrale francese. Osservatore sensibile e competente, editore perspicace e rigoroso della collana teatrale «Le Manteau d'Arlequin» di Gallimard, fin dall'inizio Lemarchand apprezza un nouveau théâtre dalle frontiere «heureusement mal définies et mouvantes» (p.33) e dalle scritture profondamente differenti. Primo sostenitore di Ionesco, convinto che «l'art est l'ennemi du repos» e che «dans l'art dramatique aussi, la monoculture peut faire des ravages» (pp. 88-89), nei suoi articoli il critico difende contro $i$ «brontosaures de toutes tendances» (Marcel Maréchal) una generazione artistica che trova nella decentralizzazione e in un'estetica nuova gli elementi fondamentali di una rivoluzione drammaturgica che si oppone a «un certain théâtre qui négligeait absolument l'homme et sa situation dans le monde» (p. 33). «Un jour, il faudra réunir ces chroniques en un livre: on y trouvera l'histoire du théâtre depuis la guerre» scriveva nel 1974 Jean Duvignaud, rendendo così omaggio al ruolo egemone ricoperto nel dopoguerra da questo «témoin équitable» (R. Abirached) poco influenzabile dalle mode letterarie (cfr. tra gli altri il testo L'Avant-garde n'est pas née d'hier, pp. 413). Tra le migliaia redatti da 
Lemarchand, la curatrice ripropone articoli rivelatisi decisivi per il successo di pièces oggi considerate come dei classici contemporanei (La Cantatrice chauve, L'Invasion, Les Bonnes, En attendant Godot, Capitaine Bada, per limitarci a pochi titoli) ma poco apprezzate al momento della loro creazione e di cui il lettore può cogliere il progressivo mutamento di ricezione grazie alla presentazione non solo della generale ma anche delle riprese che hanno avuto luogo fino al 1968. Risulta inoltre felice per una miglior comprensione della temperie dell'epoca e delle difficoltà del combat in corso la scelta di completare gli scritti professionali del critico con estratti della corrispondenza intercorsa tra quest'ultimo e autori, attori o colleghi.

2 Di piacevole e interessante lettura, questo volume costituisce così non solo un omaggio a una figura essenziale della storia del teatro francese del dopoguerra, ma anche una testimonianza importante della difficile ma progressiva affermazione della rivoluzione drammaturgica in corso durante un ventennio «tout aussi brillant pour le théâtre français que la période classique» (Marie-Claude Hubert). Va reso merito a Lemarchand - insignito peraltro nel 1971 del Grand Prix National du Théâtre - di aver compreso e sostenuto, spesso isolato assieme a poche altre voci lungimiranti, un teatro «périodiquement percé de coups pour la raison même qu'il est d'aujourd'hui» (p. 45) ma promesso a un grande avvenire. 